\title{
The vascular basement membrane in brain metastasis
}

An assumption prevails that colonization by brain metastases requires an initial interaction with neural tissue, but the literature lacks supportive evidence. Researchers from the Medical Research Council/Cancer Research UK, Gray Institute for Radiation Oncology and Biology, University of Oxford, UK, directly investigated this hypothesis, and found that "brain metastases are initiated upon cerebral vessels and not within the neural elements as has long believed to be the case," according to Shawn Carbonell, the lead author of the study.

\section{$\mathbf{4}$...brain metastases are initiated upon cerebral vessels and not within the neural elements... 77}

The incidence of secondary tumors in the brain is considerably greater than the incidence of primary brain tumors, and is on the increase owing to improved survival of patients with cancer. Coupled with a poor prognosis, these metastases represent an important therapeutic challenge to clinicians. Consequently, it is perhaps a surprise that secondary brain tumors are, in the words of Carbonell, "horribly understudied", and that relatively little is known about how they infiltrate the brain and proliferate.

The researchers found that, following parenteral injection in animal models of brain metastasis, tumor cells selectively associated with brain vasculature, a feature that was also demonstrated to characterize human brain metastasis specimens. This association with the blood vessels was determined to be adhesive in nature and required direct attachment to the vascular basement membrane of the vessel walls. This interaction seems to be the only requirement for early microcolony formation, indicating that angiogenesis is not necessary for initial growth of brain metastases. Most interesting, however, was the finding that this interaction required the $\beta 1$ integrin subunit. The use of integrin subunit-blocking antibodies considerably blocked the adhesion of tumor cells to blood vessels in brain slices, and markedly attenuated colony formation in vivo. Thus, the study seems to have identified a molecular mechanism that tumor cells use to establish colonies in the brain.

More research is required before the development of brain metastases is completely understood. The authors believe, however, that the current findings

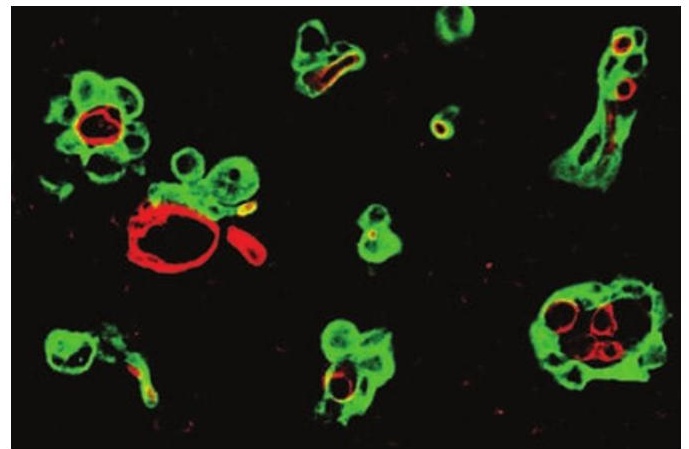

Figure 1 | Human brain metastasis demonstrating association of tumor cells (green) with blood vessels (red). Image provided by Dr Shawn Carbonell.

provide a firm foundation for further investigation in this field. As Carbonell points out, "the requirement for integrinmediated vascular co-option in brain metastasis establishment is very exciting from a therapeutic standpoint". If future studies follow up this line of research these latest findings could aid the discovery of effective molecular therapies for patients with this predominantly fatal condition.

Nick Jones

Original article Carbonell, W. S., Ansorge, 0., Sibson, N. \& Muschel, R. The vascular basement membrane as "soil" in brain metastasis. PLoS One 4, e5857 (2009). 